\title{
Gender verification: a concept whose time has come and passed?
}

Gender verification was hardly an issue at the inception of the ancient Olympic games in Greece. Competition was restricted to men, and the competitors entered the stadium unclad. No doubt existed about their gender. However, with the addition of women to Olympic competition in 1912, concerns were raised about the possibility of men attempting to impersonate female competitors for the purpose of gaining unfair advantage. In an effort to allay these fears, sex testing (now known as gender verification) was introduced in the late 1960 s to guarantee a level playing field.

The first tests, which were conducted at the European Track and Field Championships in 1966 and the Pan American Games in 1967, resembled those employed at the ancient Olympic games-women paraded unrobed in front of a panel of physicians who confirmed their gender. Because of the humiliating nature of these tests, an objective measure of gender verification, the sex chromatin test, was introduced at the Mexico City Olympic Games in 1968. This consisted of examining cells from a smear taken from the buccal mucosa of female competitors for the presence or absence of a Barr body. This body is formed after inactivation of one of the two $\mathrm{X}$ chromosomes in female cells. The presence of a Barr body confirmed the female gender of a competitor, while its absence raised suspicion about the athlete's gender. The medical code provided for karyotyping and/or a physical examination by a member of the International Olympic Committee (IOC) Medical Commission under such circumstances.

Unfortunately, many female competitors were unfairly disqualified by this test. Only the chromosomal or genetic sex of the competitor can be determined by this test and therefore phenotypic females with male sex chromatin patterns seen in conditions such as androgen insensitivity syndrome or $\mathrm{XY}$ gonadal dysgenesis are potentially subject to disqualification. While physical examination and careful review would usually result in a correct decision about the eligibility of these athletes, most women with abnormal sex chromatin tests "retired" or opted to forgo further assessment to be spared potential publicity and public scrutiny. In fact, pooled data have shown that 13 women have been excluded from athletic competition between 1972 and 1990 using sex chromatin testing: about one in every 500 female competitors. ${ }^{1}$

Sex chromatin analysis fell out of favour of most geneticists shortly after it was instituted by the IOC. The test was highly susceptible to human error, and false positive and false negative results were not uncommon. In an effort to circumvent these problems, a new gender verification test was implemented by the IOC at the 1992 Albertville and Barcelona Olympic Games. Championed by Dingeon, ${ }^{2}$ this test used polymerase chain reaction (PCR) to amplify DNA from buccal cells so that the SRY gene, a testis determining gene, could be detected on the Y chromosome.
In this issue of the journal, Serrat and Garcia de Herreros further our knowledge about this test by describing the protocol which they used during the 1992 Barcelona Olympic Games. ${ }^{3}$ Their findings of incongruity between expression of SRY and DYZ1 sequences on the $Y$ chromosome and the higher likelihood of finding DYZ1 sequences in female athletes as opposed to unselected female controls further our understanding of the technical limitations of this test. However, no matter how technically sophisticated we become in using this test, the major problems introduced by attempting to verify the female gender of athletic competitors are not resolved.

Even if we accept Dingeon's contention that PCR testing is $99 \%$ accurate, ${ }^{2}$ the possibility exists that seven of the approximately 700 females and 20 of the 2000 females who compete in winter and summer Olympic games, respectively, could be falsely positive. Fortunately, the careful work of Serrat and Garcia de Herreros ${ }^{3}$ would suggest that did not happen in Barcelona. But this begs the larger question-do we need gender verification at all?

In perhaps the most careful analysis of this issue to date, the International Amateur Athletic Federation (IAAF) brought together experts from the fields of medical genetics, gynaecology, paediatrics, endocrinology, pathology, psychiatry, and sports medicine to deliberate this issue in Monte Carlo in 1990, with a subsequent meeting of the second IAAF Working Group in London in 1992. ${ }^{45}$ The consensus was overwhelmingly in favour of abandoning gender verification.

This makes eminent sense. Laboratory testing can never circumvent the issue of phenotypic females who have male sex chromatin patterns but who have no significant athletic advantage. The issue of men masquerading as female competitors is laughable given the requirement for direct observation of athletes providing urine specimens from their urethras for drug testing and the widespread fashion statements made by athletes in their tight, body conforming spandex outfits. Indeed, I think it is reasonable to proclaim that gender verification is a concept whose time has come and passed.

JAMES C PUFFER

Los Angeles, California, USA

1 Ferguson-Smith MA, Ferris EA. Gender verification to sport: the need for change? Br 7 Sports Med 1991;25:17-21.

2 Dingeon B, Hamon P, Robert M, Schamasch P, Pugeat M. Sex testing at the Olympics. Nature 1992;358:447.

3 Serrat A, Garcia de Herreros A. Gender verification in sports by PCR-amplification of SRY and DYZ1 Y-chromosome specific sequences presence of DYZ1 repeat in female athletes. $\mathrm{Br} f$ Sports Med 1996;30:310-12.

4 Ljungqvist A, Simpson J. Medical examination for health of all athletes replacing the need for gender verification in international sports: the International Amateur Athletic Federation Plan. $\mathscr{f} A M A$ 1992;267:85052.

5 Simpson J, Ljungqvist A, de la Chapelle A, Ferguson-Smith M, et al. Gender verification and the next Olympic games. $¥ A M A 1993 ; 269: 357-8$. 